\title{
Modèles d'affaires, logistique et innovation : le cas du
}

\section{e-commerce}

\author{
Business Models, Logistics and Innovation: the case of e-commerce
}

\author{
Christine Belin-Munier ${ }^{1}$ \\ ${ }^{1}$ Université de Bourgogne Franche-Comté, Laboratoire ThéMA, UMR 6049, christine.munier@u-bourgogne.fr
}

\begin{abstract}
RÉSUMÉ. Les modèles d'affaires ont d'abord été utilisés pour les entreprises en lien avec Internet. La gratuité courante sur Internet a obligé les entreprises à se questionner sur leur modèle de revenu. La nouveauté des offres a posé la question de l'évaluation par le business plan traditionnel et engendré un nouvel outil d'analyse : le modèle d'affaires. Le modèle d'affaires est donc à la fois une pratique et un outil d'analyse. II ne remplace pas le diagnostic stratégique mais permet une autre formalisation des choix issus du diagnostic en mettant l'accent sur la création de valeur, la capture de cette valeur et les activités et acteurs à l'origine de cette création. Les modèles d'affaires peuvent être sources d'innovation, par leurs composants et les interactions entre ces composants. Nous nous proposons ici de réfléchir sur l'innovation par les choix de positionnement de la logistique et du Supply Chain Management dans les modèles d'affaires des e-commerçants.

ABSTRACT. Business models were first used for companies with an Internet connection. The current free access on the Internet obliged companies to question their income model. The novelty of the offers raised the question of evaluation by the traditional business plan and engendered a new tool of analysis: the business model. Thus the business model is both a practice and an analysis tool at the same time. It does not replace strategic diagnosis but allows another formalization of choices stemming from the diagnosis by putting accent on value creation, the capturing of this value and activities and actors at the origin of this creation. Business models can be sources of innovation, by their components and the interactions between these components. We suggest here to think about innovation by the choices of positioning of the logistics and Supply Chain Management in the business models of e-retailers.

MOTS-CLÉS. e-commerce, modèle d'affaires, business models, SCM, logistique, innovation.

KEYWORDS. e-commerce, Business Models, SCM, Logistics, Innovation.
\end{abstract}

\section{Introduction}

Les modèles d'affaires ou business models (BM) font l'objet d'un nombre important de travaux. Zott et al. (2011) dénombrent 1177 articles dans des revues scientifiques depuis 1995. 28800000 résultats sur le moteur de recherche Google, 532000 résultats sur Google Scholars, 15699 résultats sur EBSCO (Business Source Premier) : le terme est donc largement utilisé en dehors et dans le monde académique.

La diffusion des BM coïncide avec la révolution numérique dans l'économie des années 1990 (Osterwalder et al., 2005). Cette révolution a impacté de nombreux secteurs et notamment les caractéristiques du marché. Pour Teece (2010), d'une logique poussée par l'offre, beaucoup de secteurs ont dû passer vers des systèmes plus centrés sur les clients. Il ne faut plus seulement réfléchir à l'offre au consommateur mais aussi à comment capter une part de la création de la valeur alors que par ailleurs, le consommateur associe internet à la gratuité des services, et que se développent de nouveaux procédés de piraterie.

Da Silva et Trkman (2014) ont fait le parallèle entre le nombre de publications et l'évolution de l'indice Nasdaq fortement influencé par les entreprises de la net-économie. Pour eux, les modèles d'affaires ont dû être utilisés par les investisseurs pour évaluer les nouvelles entreprises intervenant sur Internet, faute d'historique utilisable. Le BM est ensuite sorti de la sphère internet pour se diffuser dans les autres secteurs économiques et même en dehors du monde des affaires, pour diverses formes d'organisations (Da Silva et Trkman, 2014). 
Un usage trop intensif du terme a induit un questionnement sur sa pertinence, avec notamment la critique de Porter (2001) qui l'oppose à la stratégie. Des travaux de recherche vont alors chercher à le définir de façon plus rigoureuse, à en cerner les différents composants (Wirtz et al., 2016). BadenFuller et Mangematin (2013) ne voient pas les modèles d'affaires seulement comme des phénomènes réels mais aussi comme des outils de compréhension permettant d'appréhender les liens de causalité entre des éléments internes et des éléments externes de l'entreprise. S'ils ne dispensent pas les entreprises d'un diagnostic stratégique (Porter, 2001), ils permettent néanmoins de renouveler la façon de voir la pensée stratégique (Lecocq et al., 2010) et de la formuler (Osterwalder et Pigneur, 2010).

Ces BM font l'objet d'une recherche majoritairement européenne et dans la sphère du management, avec trois thèmes dominants qui sont pour Lambert et Davidson (2013) la classification des entreprises, le lien avec la performance et l'innovation. Pour Zott et al (2011) les thématiques récurrentes sont plutôt l'utilisation des technologies de l'information, les questions stratégiques et l'innovation. L'innovation apparait comme une question de recherche commune dans les deux travaux. L'innovation doit s'appuyer sur des BM (Chesbrough, 2010) mais les BM eux-mêmes peuvent être source d'innovation (Teece, 2010; Amit et Zott, 2012). De ces travaux il ressort que les modèles d'affaires sont un outil d'analyse de l'innovation.

Les modèles d'affaires, en mettant l'accent sur les choix à un instant $t$ issus de l'orientation stratégique de l'entreprise (Da Silva et Trkman, 2014 ; Casadesus-Masanell et Ricart, 2010) peuvent servir de support à des taxonomies (Baden-Fuller et Morgan, 2010 ; Lambert et Davidson, 2013). Si des travaux de classification ont été d'ores et déjà réalisés (Chesbrough, 2007; Benson-Rea et al., 2013 ; Camisón et Villar-López, 2010), ils sont néanmoins difficiles à comparer et à combiner en raison d'une grande diversité d'approches. La variété de ces classifications peut s'expliquer par des clés d'analyses différentes d'un auteur à un autre. Nous proposons ici de partir d'un ensemble de composants des BM faisant le plus consensus entre les différents travaux de recherche sur la question. Cette analyse par les composants des BM permet de chercher des éléments de différenciation des choix stratégiques entre des entreprises concurrentes, résultant d'une nouvelle approche de la création de valeur par l'entreprise (innovation par le BM).

L'utilisation d'internet associée au commerce permet de diffuser une plus grande information vers un nombre plus grand de clients, dispersés sur des zones géographiques de plus en plus étendues. Si le processus d'achat peut être en grande partie numérisé, la délivrance du produit (sauf exception des produits culturels ou imprimables à distance) reste physique et sous-entend donc une définition de l'offre du e-commerçant avec un certain niveau de service logistique, des activités logistiques intégrées ou sous-traitées, un pilotage inter organisationnel de ces activités. Or la logistique et le Supply Chain Management (SCM) restent en général absents des études sur les BM, même pour le terrain du ecommerce dont la logistique constitue un enjeu stratégique tant par les coûts que par la différenciation et la focalisation. Par ailleurs la logistique et le SCM ont dans le passé été à l'origine de nombreuses innovations au niveau des produits offerts (produits facilement transportables, niveau de service logistique) et au niveau des processus (JIT, kanban, postponement, EDI, crossdocking, ...). Nous nous proposons donc ici d'étudier la stratégie d'innovation des e-commerçants à travers leurs modèles d'affaires et en particulier la place qu'y occupent les ressources et compétences logistiques et SCM (innovation par les choix de positionnement de la logistique et du SCM dans le BM).

Nous présenterons les composants du BM dans la littérature académique, le lien entre BM et innovation et la place que peut y prendre le SCM dans une première partie et appliquerons cette analyse au e-commerce dans une seconde partie. 


\section{Les modèles d'affaires}

\subsection{Définitions et composantes}

Les définitions des modèles d'affaires diffèrent beaucoup d'un auteur à un autre. La revue de la littérature académique de Zott et al. (2011), analysant une sélection de 103 publications entre janvier 1975 et décembre 2009, fait apparaître que le BM n'est pas toujours défini par les auteurs qui le mobilisent (dans 37\% des cas) et que seuls 19\% se réfèrent explicitement à une définition d'un autre auteur. De cela résulte une grande variété de définitions du BM : il est une déclaration, une description, une représentation, une architecture, un outil conceptuel, un modèle structurel, une méthode, un cadre, un patron, un ensemble. Cependant, derrière cette variété conceptuelle en partie expliquée par les silos disciplinaires des chercheurs, les auteurs distinguent des points de consensus comme une nouvelle unité d'analyse, une approche systémique, le rôle important des activités, de la création de valeur et de la capture de la valeur. Le BM semblerait plus facile à définir par ses composantes et ses caractéristiques que par son essence.

Pour Wirtz et al. (2016), la variété s'explique par une évolution dans le temps des mobilisations du BM. Si le champ d'application était très étroit à l'origine, il n'a cessé de s'étendre depuis. Le BM a d'abord été appliqué à une petite partie de l'entreprise, puis à l'entreprise pour aujourd'hui se diffuser à la situation concurrentielle de l'entreprise ; à vocation initiale très opérationnelle (pour le management des processus par les spécialistes des technologies de l'information puis pour une présentation intégrée de l'organisation dans le cadre du e-business), le BM est devenu un outil d'abstraction permettant la représentation de l'architecture d'une entreprise, pour finalement prendre ces dernières années une dimension plus stratégique et un moyen d'implanter une stratégie cohérente; les divergences s'expliquent en partie par les silos de recherche déterminés par des orientations plus tournées sur les technologies, les théories des organisations ou la stratégie. C'est cette perspective stratégique que nous allons retenir ici pour notre étude.

L'analyse de 69 recherches empiriques entre 1996 et 2010 de Lambert et Davidson (2013) fait entre autres ressortir les BM comme des outils pour analyser et communiquer les choix stratégiques, une manifestation de la stratégie, un moyen d'articuler la création de valeur, les sources internes d'avantage concurrentiel et la capture de la valeur. Ils s'appliquent à l'entreprise individuelle mais aussi aux réseaux. Le BM ne se substitue pas à la stratégie mais apparait plus comme un outil soutenant la décision stratégique en la rendant en partie visible. Pour Da Silva et Trkman (2014) les BM représentent une combinaison spécifique de ressources qui par le biais de transactions génèrent de la valeur pour le client et l'organisation. La stratégie, dans une perspective de long terme, met en place des capacités dynamiques qui viennent contraindre les possibilités des BM dans un horizon de moyenterme. La stratégie reflète ce que l'entreprise souhaite devenir alors que les BM décrivent ce qu'elle est à un instant donné. Les BM ne remplacent pas la stratégie (Porter, 2001), mais révèlent les choix des dirigeants (Casadesus-Masanell et Ricart, 2011). Ils doivent être alignés avec les buts de l'entreprise et robustes contre l'imitation, la capture de valeur ou de pouvoir, la substitution. La stratégie prévoit comment créer une position unique et valorisable impliquant un ensemble d'activités distinctives, alors que le BM reflète la logique de l'entreprise : comment elle opère pour créer et capturer de la valeur pour ses parties prenantes dans un environnement concurrentiel.

Le BM étant un ensemble de choix découlant de la stratégie, il devient une manifestation en partie visible d'une stratégie le plus souvent opaque pour la concurrence et certaines parties prenantes. Il nous paraît donc être un outil pertinent pour explorer la différenciation stratégique entre des entreprises concurrentes.

La référence la plus citée par Google Scholars est l'ouvrage d'Osterwalder et Pigneur (2010), qui est aujourd'hui largement utilisé par les accompagnateurs de la création d'entreprise en France. Selon les deux auteurs, un BM décrit les principes selon lesquels une organisation crée, délivre et capture de la valeur. Osterwalder et Pigneur (2010) proposent comme langage commun pour la description, la 
visualisation, l'évaluation et le changement de BM le modèle Canvas avec 9 blocs : les segments de clientèle, la proposition de valeur, les canaux, les relations avec le client, les flux de revenus, les ressources clés, les activités clés, les partenaires clés et la structure de coûts. L'avantage de cette approche est de questionner les choix stratégiques par 9 biais différents, mais ces blocs peuvent selon nous être regroupés en trois grands ensembles, facilitant une vision plus holistique de la stratégie. Une synthèse de 18 articles écrits entre 1998 et 2016 portant sur la définition des composants des BM nous a permis en effet d'identifier les composantes suivantes, de façon assez récurrente.

- La valeur créée pour le consommateur (mais aussi les parties prenantes, Morris et al., 2006) ou l'utilisateur de l'offre proposée par l'entreprise ; les technologies et produits intégrés à l'offre peuvent participer à cette valeur créée ; les caractéristiques de la délivrance par le niveau de service peuvent également être associées tout comme les canaux d'Osterwalder et Pigneur, 2010 ; la proposition de valeur s'adresse à un segment de clientèle, et inclus la forme de la relation que l'entreprise va mettre en place avec le client(Osterwalder et Pigneur, 2010) ${ }^{1}$.

- Les différents revenus créés (dont les incitations au paiement d'une partie de la valeur par le consommateur) et leur appropriation et donc les bénéfices financiers pour les différents acteurs de la chaîne de valeur. Ces revenus sont à mettre en rapport avec les différents coûts pour comprendre la capture de la valeur créée qui sera partagée entre les différents acteurs à l'origine de la création de l'offre et le client final. ${ }^{2}$

- Les différentes activités nécessaires à la proposition de valeur et les ressources internes et externes mobilisées pour mener à bien ces activités (Osterwalder 2004, 2005; Osterwalder et Pigneur, 2010) et donc les différents acteurs ; celles-ci définissent la structure de la chaîne de valeur et la position de l'entreprise dans cette chaîne de valeur (Chesbrough, 2007).

Certains auteurs ajoutent à ces composantes en grande partie communes les technologies utilisées (Teece, 2010), le système d'apprentissage (Itami et Nishino, 2010) et les boucles retour entre les différentes composantes (Achtenhagen et al., 2013). D'autres considèrent les interactions comme une caractéristique du BM (Plé et al., 2010) mais non une composante. Nous adopterons ce point de vue pour le reste de cette étude. Ainsi pour Wirtz et al. (2016), le BM est une représentation simplifiée des activités pertinentes d'une entreprise, qui décrit comment la valeur est créée, comment l'entreprise tire un avantage concurrentiel, le tout dans une perspective dynamique. Technologie et système

\footnotetext{
${ }^{1}$ Cette proposition de valeur ou valeur créée est explicite chez Osterwalder (2004 et 2005), Osterwalder et Pigneur (2010), Morris et al.. (2005), Chesbrough (2007), Teece (2010), Moingeon et Lehmann-Ortega (2010), Baden-Fuller et Mangematin (2013), BadenFuller et Haefliger (2013), Demil et al.. (2014), Demil et Lecocq (2010), Wirtz et al.. (2016) ; elle apparaît comme la recherche de la satisfaction ou le bénéfice du consommateur chez Timmers (1998), Amit et Zott (2012), Zott et Amitt (2010), Achtenhagen et al.. (2013) ou encore la fourniture de produits ou de services (Itami et Nishino, 2010) ou simplement par le degré de diversification (Camison et Villar-Lopez, 2010)
}

\footnotetext{
2 Timmers 1998, Osterwalder (2004 et 2005), Osterwalder et Pigneur (2010), Morris et al.. (2006), Chesbrough (2007), Teece (2010), Itami et Nishino, 2010), Camison et Villar-Lopez (2010), Amit et Zott (2012), Zott et Amitt (2010), Moingeon et Lehmann-Ortega (2010), Baden-Fuller et Mangematin (2013), Achtenhagen et al.. (2013), Demil et al.. (2014), Demil et Lecocq (2010), Wirtz et al.. (2016) ; Baden-Fuller et Mangematin (2013), Baden-Fuller et Haefliger (2013) parlent plutôt de monétisation et d'opportunités de complémentarité des actifs.
}

\footnotetext{
${ }^{3}$ Elle dépend des choix réalisés en matière intégration verticale et de collaboration (Camison et Villar-Lopez, 2010), de division du travail (Itami et Nishino, 2010) ; Amit et Zott, 2012 ; Zott et Amit, 2010) parlent de structure et de gouvernance du système d'activité ; Moingeon et Lehmann-Ortega (2010) d'architecture de la chaîne interne et de la chaîne externe ; Achtenhagen et al. (2013) de business architecture pour les flux de produit, de services, et d'informations et la description des participants au marché ; Baden-Fuller et Mangematin (2013), Baden-Fuller et Haefliger (2013) de flux d'informations et de système de gouvernance ; Demil et al. (2014), Demil et Lecocq (2010) de ressources et compétences et d'organisation interne et externe et Wirtz et al. (2016) de compétences et d'actifs, de réseaux et de partenaires, de modèle de fabrication et de modèle d'approvisionnement. Plé et al. (2010) mettent l'accent sur le consommateur lui-même comme acteur et ressource du BM.
} 
d'apprentissage peuvent quant à eux être intégrés aux activités et ressources ou à la caractérisation de l'offre.

La stratégie concurrentielle à l'origine de l'avantage concurrentiel (ou encore la stratégie marketing des acteurs de Timmers 1998, la façon d'aller sur le marché de Teece, 2010) peut être explicitement intégrée au BM (Morris et al., 2006 ; Chesbrough, 2007 ; Wirtz et al., 2006) ou à l'origine du BM qui devient le moyen de la réaliser ou son révélateur (Itami et Nishino, 2010). C'est cette seconde approche que nous avons ici choisi.

\subsection{BM et innovations}

Si durant l'ère industrielle il suffisait pour capturer la valeur d'une innovation de l'intégrer dans un produit et de le vendre, aujourd'hui l'innovation requiert le bon BM (Chesbrough, 2010 ; Teece, 2010), différencié pour assurer un avantage concurrentiel (Teece, 2010). Pour Chesbrough (2007), les coûts de développement de nouveaux produits ayant très fortement augmenté ces dernières années alors que par ailleurs la durée de vie des produits diminue, l'innovation aujourd'hui doit inclure des modèles d'affaires et ne pas se limiter à la technologie. Une technologie médiocre avec un bon modèle d'affaires peut même être préférable à une bonne technologie mais avec un médiocre modèle d'affaires (Chesbrough, 2010).

Pour Chesbrough et Rosenbloom (2002), la valeur de l'innovation demeure latente jusqu'à sa commercialisation par un BM familier ou nouveau. Le BM convertit les caractéristiques et potentiels technologiques en produits économiques à travers les consommateurs et les marchés. Il doit dans un premier temps être expérimenté de façon interne et isolée avant d'être déployé (Chesbrough, 2007). Une partie de sa composition peut être intuitive et tacite; l'expérimentation et l'apprentissage permettant sa définition plus complète (Teece, 2010).

Couplé à une analyse de la stratégie concurrentielle il permet un avantage concurrentiel durable en bloquant en particulier les imitations par les concurrents. Le BM concurrentiel durable suppose des processus et actifs difficiles à répliquer, l'opacité et un risque de cannibalisation pour les BM existants des concurrents (Teece, 2010).

Le modèle d'affaires peut par lui-même être source d'innovation (Teece, 2010 ; Amit et Zott, 2012) et d'avantage concurrentiel s'il est suffisamment différent de ceux des concurrents et difficile à reproduire (Teece, 2010). L'innovation par le BM peut se faire entre autres par l'ajout de nouvelles activités, par une nouvelle façon de lier les activités existantes, par le changement de parties qui réalisent des activités (Amit et Zott, 2012).

Le BM repose sur des hypothèses implicites concernant les consommateurs, les revenus, les coûts, le changement des usages et des besoins, les réponses prévisibles des concurrents (Teece, 2010). Les BM doivent donc être reconfigurés avec les changements de marché, de technologies et des contraintes légales. S'appuyant sur la théorie des ressources et celle des compétences dynamiques, Schneider et Spieth (2013) distinguent le développement du BM de l'innovation. L'innovation par BM est une réponse au changement des sources créatrices de valeur; le développement de BM identifie les potentiels en termes d'amélioration et d'innovation continue. Les BM doivent anticiper les changements de courbe de vie des technologies (Nunes et Breene, 2011).

L'innovation comme composante du BM permet enfin une typologie en 6 étapes de maturité : BM indifférencié, BM en partie différencié, BM segmenté, BM ouvert sur l'extérieur, BM intégrant ses processus d'innovation, BM comme plateforme adaptative (Chesbrough, 2007).

Dans un contexte où les innovations de produits sont coûteuses, longues à réaliser, déjà nombreuses et efficientes, l'innovation par le BM apparaît comme une alternative sous-utilisée, difficile à imiter et reposant sur une large analyse de la concurrence (Amit et Zott, 2012). La création de valeur dépendra 
de la nouveauté, du verrouillage (par des coûts de transfert et des incitations), par les complémentarités et l'efficience (Amit et Zott, 2012). L'innovation peut également se faire par une nouvelle offre de produits et services, par des prises de décisions retardées ou décalées (pour la fixation du prix par exemple), par le choix du décideur le plus pertinent, par l'alignement des objectifs des différents acteurs (Girotra et Netessine, 2014).

\subsection{Limites des classifications existantes des BM}

Pour Lambert (2015) la classification est cruciale pour comprendre une réalité objective. Elle implique d'ordonner des objets en groupes ou classes sur la base de leurs similarités et ordonner des objets en classes donne du sens à la réalité. C'est une étape nécessaire pour comprendre un domaine de recherche. Les catégories sont dérivées soit d'une approche conceptuelle à partir d'un petit nombre de caractéristiques et d'une démarche plutôt qualitative (typologie) soit d'une approche empirique à partir de nombreuses caractéristiques et d'une approche plutôt quantitative (taxonomie). La typologie ne peut être généralisée que de façon limitée mais comme toute approche qualitative, elle a sa place selon nous dans une approche exploratoire.

Des chercheurs ont d'ores et déjà cherché à classifier les entreprises sur la base de leurs BM. Selon Chesbrough (2007) par exemple, le cadre apporté par les BM permet de définir six types d'entreprises des BM les plus basiques aux BM les plus avancés. Les modèles les plus basiques se différencient par l'offre (indifférenciée, peu différenciée, ou segmentée). Les modèles les plus avancés sont ceux qui incluent des processus d'innovation, plus ou moins ouverts aux acteurs extérieurs à l'entreprise (fournisseurs et clients) et plus ou moins interactifs (modèle ouvert, intensification des échanges ou plateforme adaptative). Benson-Rea et al. (2013) proposent quant à eux une classification centrée sur la gouvernance, entre deux «ideal types » qui sont l'internalisation pure et l'externalisation pure du système de valeur, avec trois types mixtes. Le degré d'intégration combiné entre autres au degré de centralisation et aux mécanismes de coordination permet à Camisón et Villar-López, (2010) de définir 4 types de modèles : multidivisionnel, intégré, hybride (peu centralisé, processus et résultats normalisés très intégré verticalement) et basé sur le réseau (très centralisé, normalisation des résultats et peu intégré verticalement). Ces classifications correspondent à des approches très spécifiques centrées sur le degré d'ouverture du processus d'innovation pour Chesbrough (2007) et les relations entre acteurs (Chesbrough (2007) ;Benson-Rea et al. (2013) ; Camisón et Villar-López, (2010)).Elles ne tiennent pas compte de la création ni de la capture de la valeur qui peuvent pourtant interagir avec ces relations entre acteurs. Par ailleurs, la logistique et le SCM sont peu présents. La supply chain est seulement comprise dans le modèle d'affaires de type 5 de Chesbrough (2007). Or, pour Detchessahar et al.. (2003), la chaîne logistique est révélatrice des modèles d'affaires choisis par les entreprises : "Les supply chain donnent accès aux modèles d'affaires dans la mesure où elles traduisent la structure d'investissement du site (les activités qui sont conservées et celles qui sont sous-traitées), l'organisation du service, les stratégies concurrentielles des acteurs à l'intérieur d'une même chaîne ou entre chaîne (Detchessahar et al.. (2003) p.96). » Si les dimensions opérationnelles de la logistique et du SCM peuvent être vues comme des conséquences des BM et ainsi faire partie des choix résiduels après la détermination du BM (Casadesus-Masanell et Ricart, 2010), ce qui les exclurait des BM, ces dimensions opérationnelles sont doublées de dimensions stratégiques qui impliquent non seulement la logistique et le SCM comme des choix résiduels mais également comme des choix à l'origine de la détermination du BM. La logistique est pour Amit et Zott (2012) une activité importante du système d'activités qui est à la base du BM. Itami et Nishino (2010) et Camisón et Villar-López, (2010) évoquent le kanban en exemple de composante du BM. Une source d'innovation de rupture étant une accessibilité nouvelle d'un produit pour un segment de client (Christensen 2002 repris par Robles, 2015), la logistique a toute sa place dans la caractérisation des BM de distribution et en particulier des BM des e-commerçants. Pour Chapman et al. (2002), les prestataires de services logistiques doivent être parties prenantes dans les nouveaux BM ou paradigmes d'affaires liés aux nouvelles technologies. 
Pour Le Council of Supply Chain Management Professionnals, qui regroupent les spécialistes du monde académique et de celui des entreprises, le Supply Chain Management, qui englobe aujourd'hui la logistique, comprend la planification et le management de toutes les activités impliquées dans le sourcing et l'approvisionnement, la transformation et toutes les activités du management logistique ; il inclut aussi la coordination et la collaboration avec les partenaires du canal qui peuvent être des fournisseurs, des intermédiaires, des prestataires de services et des clients. Par essence, le SCM est une fonction intégratrice avec comme responsabilité primaire le lien des principales fonctions et principaux processus dans et entre les sociétés, dans un BM cohésif et très performant (cohesive and highperforming business model). Le management logistique quant à lui planifie, met en place et contrôle le flux efficient et efficace aller et retour ainsi que le stockage de biens et services, et l'information liée depuis le point d'origine des matières première jusqu'au point de consommation pour satisfaire les attentes des consommateurs4. Le SCM se caractérise (Esper et Crook, 2014) par un contenu (des ressources et compétences), une structure (la façon de les gérer et les combiner) et une dimension stratégique (Belin-Munier, 2008). Logistique et SCM permettent entre autres de différencier l'offre de produit par une association produit/service (par la disponibilité, le délai, la fiabilité du délai, la qualité du transport, l'information sur l'avancement de la prestation, Dornier, 2007) et de modifier la structure des coûts et les relations entre acteurs à l'origine de l'offre au client final (par l'efficience du processus logistique lui-même et l'intégration de la Supply Chain, Belin-Munier, 2008).

La logistique (et le SCM) en tant que services associés au produit, comme les autres systèmes produits-services doit être intégrée dans le modèle d'affaires (Barquet et al., 2013). Or, si Barquet et al. (2013) ont bien tenté d'intégrer la maintenance et le retrait en fin de vie dans le BM de vente de matériel de production, en s'appuyant sur la structure Canvas, il n'y a pas de travaux de ce genre mené sur la logistique et le SCM. Avec les nouveaux besoins des entreprises dans un environnement hyperconcurrentiel, globalisé, bouleversé par les technologies de l'information et de la communication, la logistique doit s'appuyer sur des innovations tant technologiques qu'en matière de connaissances et de management de réseaux (Chapman et al., 2002). La logistique peut être à la fois un vecteur et une forme d'innovation par la remise en cause organisationnelle qu'elle suppose (Fabbe-Costes et Paché, 2013 ; Belin-Munier, 2011).

\section{Le e-commerce a la lumière des modèles d'affaires}

\subsection{Classifications existantes appliquées au e-commerce}

Les BM associés au e-commerce font peu l'objet de classification sur la base des composantes qui les définissent; les études sont souvent partielles (sur la connaissance, l'efficience, la création de valeur, la monétisation).

Les taxonomies relatives aux marchés électroniques (par exemple Timmers, 1998) ou aux activités du Web (par exemple Isaac et Volle, 2011 ; Rappa, 2010) reposent principalement sur une analyse de l'offre (en distinguant par exemple les e-boutiques des e-approvisionnements, des e-centres commerciaux, des places des de marché, des plateformes collaboratives, des brokers, des communautés virtuelles ou prestataires de services de Timmers, 1998). D'autres approches mêlent la création de revenu et la création d'offre (citons le modèle publicitaire, le modèle d'info-médiateur, le modèle de vente directe par le producteur, le modèle d'affiliation, le modèle communautaire, le modèle d'abonnement ou de paiement à l'utilisation de Rappa, 2010). Pour Detchessahar et al.. (2003), l'offre et la monétisation utilisées de façon croisées permettent de distinguer plusieurs modèles: le modèle industriel (faible nombre de références, pas de stock, pas de choix dans les modes de livraison internalisés, tarification unique des livraisons, forte intégration informationnelle), le modèle de service industriel-flexible (grand nombre de références, stock, choix du mode de livraison, tarification des

\footnotetext{
${ }^{4}$ http://cscmp.org/sites/default/files/user_uploads/resources/downloads/glossary-2013.pdf
} 
livraisons en fonction de la qualité de service, intégration informationnelle moyenne) et le modèle marchand (nombre moyen de références, faible niveau de stock, choix de modes de livraison externalisée, tarification des livraisons négociable, pas d'intégration informationnelle).

Marouseau en 2001 définit quatre modèles à partir de deux dimensions liées aux ressources et activités : l'utilisation d'un magasin existant ou l'investissement dans un système; la logistique des villes (avec le client qui se déplace) et la logistique des domiciles (avec les livraisons particulières). Les quatre modèles sont le modèle du store-picking, celui de l'entrepôt dédié, le modèle point-boutique et le modèle traditionnel.

Des études précédentes il découle que les approches du e-commerce par les BM sont plutôt anciennes et n'associent jamais les trois composantes que nous avons tirées de notre revue de la littérature: création de valeur, capture de valeur, activités et acteurs. Il manque une approche croisée de ces trois composantes.

\subsection{Autres travaux revus à la lumière des $B M$}

Pour compléter les travaux précédents, nous pouvons utiliser notre grille de lecture BM pour revisiter d'autres travaux portant sur le e-commerce, mettant l'accent sur une ou plusieurs de ces composantes, même s'ils ne font pas de référence explicite au BM. Les BM permettent de regrouper les travaux sur la logistique du e-commerce selon qu'ils se focalisent sur la valeur créée pour le consommateur, la capture de cette valeur avec en particulier le modèle de revenus ou les différentes activités et acteurs.

\subsubsection{La valeur créée pour le consommateur}

Pour Kessous (2001), le modèle d'affaires du e-commerce repose de plus en plus sur la personnalisation de la réponse au client, ce que les économistes appellent la qualité du produit (et les gens du marketing les attributs) ; «transposée au commerce électronique, la question de la qualité se focalise sur la prestation logistique. C'est en effet dans une réponse adaptée à la singularité du client que l'entreprise du e-commerce pourra valoriser son service, la qualité émergeant d'un processus de coproduction dans lequel intervient l'offreur mais aussi le demandeur... La logistique est le chaînon central permettant de transformer un bien standard en un produit personnalisé par cette dynamique de requalification des termes de l'échange. »(Kessous, 2001, p108).

Performance dans la réalisation de la commande (Griffis et al., 2012), fiabilité des délais (Spears et al., 2010), loyauté du client et ressources logistiques (Dadzie et al., 2005 ; Tokman et al., 2012), eservice (Field et al., 2004), satisfaction et disponibilité en stock (Taylor et al., 2004 ; Kim et Lennon, 2011), les études et thématiques sont nombreuses mais sans continuité ni lien. Elles montrent néanmoins que dans l'univers numérique du e-commerce la logistique a une part importante à jouer pour la matérialisation de la transaction. Plusieurs travaux se sont intéressés au niveau de service logistique et à son impact sur la satisfaction des clients dans le cadre du e-commerce. Citons par exemple Rao et al. (2011) qui ont étudié le lien entre la satisfaction liée à la distribution physique et la satisfaction liée aux achats à partir de données sur 260 distributeurs, Griffis et al. (2012) qui ont montré à partir d'équations structurelles le lien entre la bonne réponse à une commande, l'évaluation positive du site et l'acquisition de nouveaux clients.

Si la logistique contribue à différencier l'offre par le niveau de service, la numérisation de l'achat peut faire oublier au consommateur la partie physique de la transaction et générer des perceptions négatives de ce service. Dadzie et al. (2005) se sont intéressés au décalage qui peut exister entre la facilité d'utilisation du site, les attentes logistiques très fortes qui en découlent et donc la déception des clients par rapport à leurs attentes et le rôle de l'information pour ramener les attentes à un niveau réaliste et donc diminuer le niveau d'insatisfaction (à partir des expériences d'achats de 373 employés de deux universités américaines). 
Le marketing et la communication qu'il produit peuvent influencer la perception du service associé à l'offre en jouant à la fois sur la diversité de l'offre tout en guidant le consommateur vers des catalogues plus restreints : c'est la stratégie « podium » de Dang Nguyen et Mevel (2007). La stratégie $\mathrm{du}$ " podium » consiste à mettre en avant une sélection de produits sur la base d'une compétence de sélection spécifique du e-commerçant (Dang Nguyen et Mevel, 2007). Le e-commerçant peut alors allier la gamme étendue des références disponibles à une rotation plus importante de quelques références (celle mise en avant sur le "podium ») pour diminuer ses coûts logistiques. Ces travaux vont dans le sens de ceux de Hult et al. (2007) sur le lien entre stratégie logistique et communication : le manager doit choisir sa stratégie logistique, ajuster l'activité de l'entreprise pour qu'elle soit cohérente avec sa stratégie, communiquer vis-à-vis du client sur la valeur ajoutée de la stratégie adoptée et vérifier l'alignement de perception entre les meilleurs clients et les autres clients pour augmenter les intentions de rachats.

\subsubsection{La capture de la valeur}

Le e-commerce s'appuie sur différents modèles de revenus découlant de l'offre créée qui peut être la transaction ou la mise en relation. Pour Isaac et Volle (2011), le e-commerce repose principalement sur deux modèles d'affaires: le modèle transactionnel et le modèle relationnel. Dans le modèle transactionnel, l'entreprise vend ce qu'elle produit ou revend ce qu'elle a acheté ; dans le modèle relationnel, l'entreprise met en relation des acheteurs et des vendeurs. Dans les modèles transactionnels on trouve les sites marchands (e-tailers), les sites de vente directe par les industriels et les sites de vente privée (quantités limitées, logique de club). Les sites marchands peuvent être des pure players s'ils n'ont pas d'autre point de vente que le site internet des click and mortar s'ils ont ajouté ce mode de commerce à un mode traditionnel de point de vente physique (brick and mortar), des anciens de la vente à distance (click and paper), des sites de déstockage, des cybermarchés (épicerie en ligne), des sites horizontaux (associations de libraires), ...Dans les modèles relationnels on trouve les sites de courtage qui proposent des offres au nom de différents entreprises (produits financiers, billetterie, tourisme, ...), des infomédiaires (guides, annuaires, sites comparateurs de prix), les galeries marchandes virtuelles, les sites C2C (eBay, PriceMinister, leboncoin, Alibaba), les achats groupés, les places de marché électroniques (qui ne sont pas dans notre champ d'études). Ces modèles d'affaires s'appuient sur différents modèles de revenus : la marge commerciale brute, la commission (modèles relationnels), le revenu publicitaire (commercialisation de l'audience), le revenu d'affiliation (un site redirige une partie du trafic vers un autre) ou encore la prestation de service.

Pour le modèle transactionnel, si le consommateur attend un niveau de service associé au produit acheté (offre créée), selon Marouseau (2005), «il est généralement admis que le consommateur traditionnel n'a pas conscience du temps, de l'énergie et du coût du transport nécessaire à l'accomplissement de son achat. Le client est donc réticent à payer pour un service qu'il ne considère pas à sa juste valeur. L'acceptation par le client du prix de la livraison est donc un enjeu capital pour le développement des cybermarchés. » (Marouseau, 2005, p. 16). Le prix du service de livraison ou du service logistique pose question (Basu et al., 2004 ; Rabinovitch, 2004). Une dégradation du niveau de service peut être acceptée par un niveau de prix plus faible : un délai plus long peut faire l'objet d'un prix plus bas (Hantula et Bryant, 2005).

Concernant la structure des coûts, Rabinovich et Evers (2003) font le lien entre la localisation des stocks et les coûts; dans les travaux de recherche, le problème du dernier kilomètre de la livraison à domicile (LAD) souvent proposée en e-commerce est plus abordé sous l'angle de l'efficience et par la simulation que du point de vue de la différenciation de l'offre: Boyer et al. (2009) s'intéressent à l'impact de la densité de population et de l'amplitude de la fenêtre de livraison sur l'optimisation des tournées ; Rabinovich (2005) sur les transbordements d'urgence, la densité et les coûts de la LAD.

La capture de valeur se ferait donc plus au profit du consommateur s'il ne prend pas en charge les coûts liés à la création de valeur (liés au service logistique). Pour que le e-commerçant se réapproprie 
une plus grande part de la création de valeur, les solutions possibles à priori sont la dégradation du service justifiée par une communication à l'intention du consommateur, mais aussi la réduction de ses coûts en particulier logistiques, le transfert de la charge de ces coûts sur un autre acteur ou l'ajout d'un autre modèle de revenu, issu du modèle relationnel par exemple.

\subsubsection{Activités et acteurs}

Les activités analysées sont la gestion de stock (Kull et al., 2013 ; Taylor et al., 2004 ; Rabinovich 2003, 2004, 2011), les retours (Mollenkopf et al., 2007 ; Rao et al., 2014, Kokkinaki et al., 1999), le management des systèmes d'information (Waller and Fawcett, 2013 ; Wang, 2008, Rabinovich, 2007 ; Sanders, 2007).

Les activités nécessaires au service logistique peuvent être réalisées par le e-commerçant ou transférées, plus ou moins partiellement, comme d'autres activités habituelles du e-commerçant à un autre acteur comme le consommateur (Rouquet et al., 2010). Ce transfert sera d'autant plus simple que le consommateur a une mauvaise perception des coûts de transport. Certaines activités peuvent également être assurées par un prestataire de service logistique. Rabinovich (2004) justifie l'internalisation de la logistique en cas de spécificité des services et d'incertitude sur la réalisation de ces services (à partir de 196 questionnaires). Les travaux d'Esper et al. (2003) ajoutent le caractère stratégique du choix du transporteur avec notamment la notion de co-branding. Le transfert peut également concerner le commerçant traditionnel. Pour l'épicerie en ligne, le rôle des petits commerces indépendants peut être amené à évoluer (Durand, 2005 et 2004) dans un secteur par ailleurs regroupant trois modèles logistiques fondamentaux que sont le stockage chez les producteurs (pour les produits de luxe ou la vente locale de produits de première nécessité), le stockage chez le distributeur (avec préparation de commande en entrepôt ou en magasin), et le modèle de livraison hors domicile par le biais de centres de distribution locaux ou des points de retrait (Durand, 2008). Le drop-shipping permet de transférer une partie des activités logistiques sur le fournisseur (Cheong et al.. 2015 ; Rabinovitch et al., 2008). Au contraire, des e-commerçants peuvent faire le choix d'investir dans des capacités logistiques (Randall et al., 2006 ; Huang et al., 2012). Selon Isckia (2009), le développement des ressources d'Amazon, grâce à sa stratégie de croissance, lui a permis de proposer de nouvelles offres créatrices de valeur comme la prestation logistique.

La e-distribution (distribution associée au e-commerce), qui suppose des activités spécifiques comme la LAD, les retours, la production d'informations dédiées, peut donc relever de différents acteurs allant du fournisseur au client en passant par le e-commerçant les PSL ou encore le commerçant traditionnel.

Les études centrées sur la logistique et le e-commerce mais non issues d'approches BM montrent donc la pertinence de chacune de ces composantes pour la logistique: la logistique et le SCM permettent de différencier l'offre par le service associé, mais cette différenciation est difficile à évaluer par le consommateur final qui est de ce fait réticent à payer. Les revenus issus du modèle transactionnel ne permettent donc pas toujours au e-commerçant de capturer une part suffisante de la valeur créée. Le e-commerçant peut choisir de dégrader le service en communiquant avec le consommateur ou de transférer des activités sur d'autres acteurs comme les fournisseurs. Il peut également rechercher d'autres revenus issus de modèles plus relationnels. La e-distribution s'appuie sur des activités et ressources spécifiques comme les retours, la LAD, les technologies de l'information, le stockage. Ces activités peuvent être assumées par différents acteurs de la chaîne logistique que sont le fournisseur, le e-commerçant, le PSL, le commerçant traditionnel voire le client final. S'en suivront d'autres modèles de revenus et donc d'autres captures de la valeur mais également des besoins de pilotages spécifiques. Mais peut-on lier les choix en matière de création de valeur, de capture de valeur, d'activités et d'acteurs ? Les études précédentes ne le permettent pas, aussi avonsnous procédé à une étude qualitative exploratoire. 


\subsection{Etude de cas}

Pour notre étude de cas nous nous sommes appuyés sur des entretiens semi-directifs d'acteurs du ecommerce (10 entretiens semi-directifs de cadres et de responsables logistiques de e-commerçants et de e-logisticiens d'une durée modale d'1h30) ainsi que sur des données factuelles et déclaratives de différents témoignages de professionnels relevés dans la presse professionnelle logistique (54 dossiers thématiques) et lors de congrès et de conférences destinés aux professionnels de la logistique (journée sur le e-commerce organisée à Dauphine, le 7 décembre 2012 ; Forum des rois de la Supply Chain, le 16 janvier 2013, SITL 2013), ce qui se justifie par le caractère encore émergent de la e-logistique et l'activité réflexive des marchés économiques (Kessous, 2001).

\subsubsection{Logistique et création de valeur pour le consommateur}

Concernant la création de valeur, outre des innovations très médiatisées comme la livraison par drones ou l'impression 3D et plus anciennement le téléchargement de produits audiovisuels et l'impression à la demande d'ouvrages ou de billets, les e-commerçants ont développé ce que les professionnels du secteur appellent aujourd'hui les sites de deuxième génération, car ils offrent un choix étoffé de modes de livraison (livraison à domicile avec des délais plus ou moins courts, livraison en points de retrait, livraison en boutique de la chaîne, livraison ultra-rapide par coursiers dans les grandes villes, dépôts en consignes, ...), une traçabilité des informations à chaque étape du produit, une gestion rapide des retours permettant de fidéliser le client (et donc d'économiser les coûts de transformation d'internaute en client) et de transformer un internaute insatisfait en client fidèle. Le niveau de service est plus ou moins coûteux à atteindre selon les produits proposés à la vente, néanmoins les offres tendent de plus en plus à être similaires.

\subsubsection{Service logistique et capture de la valeur}

Les modes de récupération de la marchandise sont nombreux et les frais inhérents à la commande dépendent du mode de livraison choisi ; ils incitent au retrait en point relais ou en point de vente mais ne correspondent en général pas au vrai prix du transport et font souvent l'objet d'offres commerciales (et sont alors réduits ou gratuits pour le client). Les e-commerçants sont prêts à ajuster leurs modèles d'affaires pour des produits spécifiques (comme les produits volumineux et lourds), en intégrant un niveau de service avec un très faible sur-prix.

Ils peuvent néanmoins chercher à influencer directement ou indirectement la demande des clients vers une offre plus ou moins dégradée de niveau de service : la zone de chalandise peut être filtrée par l'intermédiaire de codes postaux; des sites communiquent sur l'impact écologique de la livraison (Monoprix et les tranches horaires de livraison); les délais et tarifs peuvent différer en fonction du mode et du délai de livraison (comme le font depuis toujours les VPCistes) ; les magasins en propre ou appartenant à un réseau de distribution peuvent servir de points de livraison et donc éviter la LAD; le client peut accepter un délai de livraison plus long en échange de l'appartenance à un cercle privilégié (vente évènementielle de Vente Privée).

Ces offres font l'objet d'expérimentations ; dans la distribution alimentaire par exemple, la LAD est en retrait par rapport à la réception de la marchandise en drive par les clients (la préparation de commande étant le plus souvent offerte).

Pour pallier la difficile monétisation de l'offre, des modèles de revenus peuvent être combinés et non plus différenciés en fonction des offres comme c'est le cas dans les BM traditionnels. Ainsi, un même site peut proposer la distribution de produits informatiques et électronique grand public sur Internet (métier initial) et une galerie marchande. Des sites réalisent peu de marge commerciale, mais tirent leurs bénéfices d'autres sources de revenus comme les revenus publicitaires. Du modèle transactionnel le site peut passer sur un modèle plus relationnel comme la vente d'évènements pour amener les consommateurs vers les marques : le fournisseur devient le client. 
La nouvelle proposition de valeur (avec le haut niveau de service logistique associé au produit) dans le e-commerce repose en grande partie sur une nouvelle capture de la valeur (au profit du client), s'appuyant sur de nouveaux modèles de revenus issus eux-mêmes de nouvelles activités dont la mise en relation mais aussi la e-distribution et de nouveaux acteurs.

La chaîne logistique, qui fait partie de la chaîne de valeur, se structure autour de plusieurs activités : la gestion de la commande (attribution d'un produit à un client), la préparation de la commande (récupération du produit physique, emballage, consolidation avec d'autres produits éventuellement), la gestion des stocks et l'approvisionnement, l'entreposage (conception et pilotage des entrepôts), l'organisation du transport, la réalisation du transport et la logistique des retours. La e-distribution repose donc sur les ressources traditionnelles de la logistique mais également sur des ressources spécifiques, qui vont devoir être développées en interne ou acquises chez des sous-traitants. La edistribution doit faire face à des contraintes spécifiques du e-commerce mais communes à tous les ecommerçants comme l'instabilité des volumes traités, l'hétérogénéité des flux, la continuité du flux des commandes, un nombre élevé de références pour un petit nombre de produits par colis, un besoin important d'informations sur le client pour la livraison et sur les produits pour les contrôles volumétriques lors du processus de préparation de commandes et l'identification lors des retours, le risque de vol des colis lors de la distribution, le volume important des déchets générés par la préparation de commandes (emballages fournisseurs), la prise en charge des produits retournés, la livraison à domicile en cas d'absence du client.

De nouvelles technologies et de nouveaux processus ont dû être mis en œuvre. La préparation est le plus souvent semi-automatisée en France : un système de convoyeurs amène le colis dans différentes gares où un opérateur effectue le picking à l'aide d'outils informatiques embarqués ou d'armoires à signaux lumineux; des systèmes automatisés adaptent la hauteur du contenant au volume de la commande. Ces actifs spécifiques dédiés supposent de gros volumes pour bénéficier d'économies d'échelle. Ces volumes peuvent être obtenus par la mutualisation de l'activité logistique avec un ecommerçant ou la prestation de services logistiques à différents clients. De nouvelles formes organisationnelles sont venues accompagner ces nouveaux processus.

La presse et nos entretiens permettent de trouver des exemples allant de l'intégration la plus complète à l'externalisation totale de ces différentes activités. Nous pouvons remarquer que si les exemples de logistique intégrée sont nombreux, il n'en n'est pas de même pour le transport final. Ce transport aval peut être sous-traité en cascade par les transporteurs eux-mêmes. La pression sur les coûts redescend alors sur le transporteur final qui est soit un sous-traitant, soit le client lui-même dans le cas de retrait dans des points de vente (en propre ou non).

Les stocks peuvent être chez le fournisseur ou chez le commerçant en fonction des types de ventes. Lorsque les stocks sont chez le e-commerçants ils sont centralisés nationalement ou au niveau européen ou localisés au plus près des points de livraison et donc décentralisés (Amazon).

Si la logistique semble source de capture de valeur pour le e-commerçant, cela semble être moins le cas du transport. Les récentes opérations d'intégration d'Amazon peuvent poser question, mais être en partie expliquée par des rapports de force spécifiques entre Amazon et ses prestataires de transport.

\subsubsection{Différenciation des BM}

Confrontées aux mêmes attentes des clients (niveau de service élevé à un faible prix), des ecommerçants peuvent donc proposer des créations de valeur similaires (variété de choix de modes de distribution du produit, avec des prix différenciés mais peu élevé voire nuls en cas d'offres promotionnelles, et pas toujours très clairs pour le consommateur), en s'appuyant sur un même 
ensemble d'activités (spécifiques à la e-distribution mais communes aux e-commerçants) mais selon des structures très diverses, faisant l'objet d'expérimentations et d'évolution.

Le niveau d'intégration des activités logistiques et SCM et le degré d'ouverture nous ont permis de mettre en évidence 9 types de structures de chaînes logistiques, sans lien univoque avec les différents modèles d'offres et de monétisation par le client, mais intégrant les activités spécifiques à la edistribution (tableau en annexe) :

-Type 1 : logistique et transport intégrés mais ouverts à d'autres e-commerçants pour de la prestation logistique (les activités réalisées en interne sont vendues comme des prestations logistiques à d'autres e-commerçants).

- Type 2 : logistique et transport intégrés mais sans ouverture à d'autres commerçants (les activités intégrées ne sont pas partagées avec d'autres acteurs ce qui limite les économies d'échelle).

- Type $3:$ logistique intégrée et transport en partie intégré pour certaines familles de produits (les activités logistiques sont intégrées, mais le transport est en partie sous-traité en fonction de la nature des produits et du savoir-faire du e-commerçant).

- Type 4: logistique intégrée et transports sous-traités avec une ouverture à d'autres commerçants (par le biais de la vente de prestations).

- Type 5: modèle différencié géographiquement et ouvert (l'intégration dépend de la zone d'implantation, et peut être vendue sous forme de prestation à d'autres commerçants)

- Type 6 : logistique intégrée mais avec l'accompagnement d'un prestataire (pour les locaux et la formation du personnel par exemple),

- Type 7 : logistique intégrée par croissance externe ou joint-venture (d'un PSL ou d'un autre ecommerçant)

-Type 8 : logistique et transport sous-traités (mais pilotés par le e-commerçant)

-Type 9 : logistique et transport sous-traités, mutualisation prestée par le sous-traitant logistique.

De nos études de cas il ressort que les composantes à l'origine d'une différenciation des BM relèvent principalement de la structure créatrice de valeur (les acteurs), même si quelques différences peuvent exister au niveau de la création de valeur (délais plus long par exemple), de la capture par le client (par le biais de facturations plus ou moins importantes de la distribution), ou encore les activités (entrepôts centralisés ou non).

Si le fournisseur peut prendre en charge les stocks et parfois le transport vers le client (pour certaines ventes de e-commerçants), la logistique est plutôt intégrée quand les volumes permettent d'amortir les frais fixes des entrepôts dédiés. Lorsqu'elle est intégrée, les économies d'échelle peuvent être renforcées par la mutualisation ou la prestation de service. Un nouveau modèle de revenu vient alors compléter le revenu transactionnel classique.

Le transport est intégré lorsqu'il est associé à la fonction commerciale et pour l'un des deux acteurs historiques de la VPC en France. Dans les autres cas, il est le plus souvent sous-traité en cascade. Dans les grandes agglomérations, le dernier kilomètre peut être pris en charge par des coursiers avec éventuellement des modes de transport doux alternatifs (vélo électrique). Rares sont les e-commerçants assurant en interne les activités en aval de la chaîne logistique. Des expérimentations tentent d'intégrer le client final dans ces activités en aval pour la prise en charge du dernier kilomètre.

\section{Conclusion}

Même si le terme de Business Model ou modèle d'affaires a fait l'objet d'usages parfois détournés et en dépit de la coexistence encore aujourd'hui de plusieurs définitions, il n'en demeure pas moins que 
l'utilisation du BM a un double apport cognitif et managérial. Il permet une autre formulation du discours stratégique et ses composantes fournissent un point de départ pour des typologies. Il permet de cerner dans les choix visibles issus du positionnement stratégique moins explicite, les éléments différenciant des entreprises concurrentes. Parmi ces composants, la logistique et le SCM ont toute leur place.

Appliqué à son domaine d'origine, le e-commerce, il permet une typologie essentiellement par les acteurs créateurs de l'offre. En effet, la création de valeur par le service logistique permet peu de différencier les e-commerçants, l'offre de référence étant une proposition hybride : plus de service à un moindre prix. La capture de cette valeur ne peut passer par une réelle monétisation du service offert, et repose donc sur la multiplication des modèles de revenus: modèles transactionnels, modèles relationnels et modèles publicitaires.

Ce sont las acteurs prenants en charge les différentes activités logistiques qui sont source de différenciation des BM. Certes les activités ont fait l'objet d'innovations technologiques, mais ces activités sont assez similaires d'un e-commerçant à un autre. La différenciation se fait plus par les acteurs les prenant en charge. Les dernières évolutions sont de nouvelles organisations permettant la mutualisation de ces activités et de nouveaux modèles de revenus en découlant. La chaîne de valeur se trouve ainsi partagée entre fournisseurs, e-commerçants, prestataires logistiques, transporteurs et clients de façon variable d'un modèle à un autre. Sous-traitance et mutualisation logistique viennent modifier le modèle de revenu transactionnel initial, et solutionner en partie le problème de la monétisation de l'offre. Si l'innovation technologique a permis la diminution des coûts logistiques, elle nécessite des volumes d'activités suffisants pour générer des économies d'échelle. Cette innovation a dû trouver son nouveau modèle d'affaires, basé sur la mutualisation et le partage des infrastructures et des compétences. Ces nouvelles pratiques organisationnelles ne prennent tout leur sens que par une approche simultanée de la création, de la capture et des activités et acteurs de la valeur.

La simple observation extérieure a cependant des limites; SCM et modèles de capture de valeur entre acteurs en dehors du client final ne sont qu'en partie dévoilés par les entretiens semi-directifs. La méthode part de présupposés conceptuels qui peuvent ignorer des éléments émergents dans un contexte de secteur nouveau et de démarche exploratoire. Il conviendrait de prolonger ce travail par d'autres approches de type longitudinal pour mieux cerner les phases d'expérimentation des BM, et des approches quantitatives dans le but de rechercher des taxonomies. Certains choix de e-commerçants peuvent en outre être un contournement temporaire de failles juridiques ou de barrières règlementaires (accès au marché parisien pour l'alimentaire, implantation sans autorisation, impôts et taxes, ..), ce qui peut fausser un temps l'interprétation.

Le BM fournit néanmoins un outil cohésif pour les différents travaux portant sur la e-distribution et permet de différencier l'innovation par l'offre (qui a permis de passer de la VAD au e-commerce de deuxième génération), par les processus (les nouvelles activités et technologies mises en œuvre pour les besoins du e-commerce) et par la chaîne de valeur (les nouveaux acteurs comme les e-commerçants prestataires logistiques, les spécialistes du dernier kilomètre en milieu difficile et les nouveaux modèles de revenus qui en découlent comme le revenu relationnel ou la rémunération de service entre entreprises).

\section{Bibliographie}

Achtenhagen L., Melin L., Naldi L. (2013), "Dynamics of Business Models -Strategizing, Critical Capabilities and Activities for Sustained Value Creation", Long Range Planning, 46, pp. 427-442

Amit R. et Zott C. (2001), "Value creation in e-business", Strategic Management Journal, vol 22, pp 493-520.

Amit R. et Zott C. (2012), "Could your company benefit from a new business model? Consider theses six questions", MIT Sloan Management Review, Spring, http://sloanreview.mit.edu/article/creating-value-through-business-modelinnovation/ 
Baden-Fuller C., Haefliger S. (2013), « Business Models and Technological Innovation », Long Range Planning, 46, pp 419-426

Baden-Fuller C., Mangematin V. (2013), «Business models : A challenging agenda », Strategic Organization, 11(4), pp 418-427

Baden-Fuller C., Morgan M.S., (2010), « Business Models as Models », Long Range Planning, 43, pp 156-171.

Barquet A.P.B., Gouvea de Oliveira M., Amigo C. R., Cunha V.P., Rozenfeld H., (2013), «Employing the business model concept to support the adoption of product-service systems », Industrial Marketing Management, vol 42, pp. 693-704

Basu A., Ingene C.A, Mazumdar T., (2004), "The pricing of delivery services”, Journal of Regional Science, vol 44, $\mathrm{n}^{\circ} 4$, pp. $743-772$

Belin-Munier C, (2011), "L'innovation dans les chaînes logistiques, Le Supply Chain Management vecteur d'innovation pour les PME ? », dans PME, dynamiques entrepreneuriales et innovation, Hamdouch A, Reboud S, Tanguy C (dir), PIE Peter Lang, pp 303-323

Belin-Munier C., (2008), Etat de la recherche sur le Supply Chain management et sa performance : une revue de la littérature récente, Logistique \& Management, vol 16, n², pp 17-29

Benson-Rea M., Brodie R., Sima H., (2013), "The plurality of co-existing business models : investigating the complexity of value drivers", Industrial marketing management, 42, pp 717-729

Boyer K.K., Prud'homme A.M., Chung W., (2009), « The last mile challenge : evaluating the effects of customer density and delivery window patterns", Journal of business logistics, vol 30, n 1 , pp. 185-201

Camisón C. et Villar-López A., (2010), «Business Models in Spanish Industry : a Taxonomy-based Efficacy Analysis », M@n@gement, vol 13,nº,pp.298-317

Casadesus-Masanell R., Ricart J. E., (2010), "From Strategy to Business Models and onto Tactics", Long Range Planning, 43, pp. 195-215

Chapman RL, Soosay C., Kandampully J., (2002), "Innovation in logistic services and the new business model: a conceptual framework”, Managing Service Quality: An International Journal, vol 12, n², pp. 358-371

Cheong T., Goh M., Song S. H., (2015), "Effect of inventory information discrepancy in a drop-shipping supply chain", Decisions Sciences, vol 46, number 1, pp. 195-210

Chesbrough H., (2010), « Business Model Innovation : Opportunities and Barriers », Long Range Planning, 43, pp. 354363

Chesbrough H., (2007), « Business model innovation : it's not just about technology anymore », Strategy \& Leadership, vol 35, n6, pp 12-17

Chesbrough H., Rosenbloom R. S., (2002), "The role of the business model in capturing value from innovation: evidence from Xerox Corporation's technology spin-off companies”, Industrial and Corporate Change, vol 11, number 3, pp. $529-555$

Christensen C.M., Johnson M.W., Rigby D.K., (2002), «Foundations for growth : how to identify and build disruptive new businesses », MIT Sloan Management Review, 43(3), pp 22-31

DaSilva C. M., Trkman P., (2014), «Business Model : What It is and What It is Not », Long Range Planning, vol 47, pp 379-389

Dadzie K.Q., Chelariu C., Winstan E, (2005), « Customer service in the internet-enabled logistics supply chain : webside desgn antecedents and loyalty effects », Journal of business logistics, vol 26, n 1 , pp 53-78

Demil B., Lecocq X., Warnier V., (2014), "Business models”, dans Encyclopédie de la stratégie, coord par Tannery F, Denis JP, Hafsi T et Martinet AC, Vuibert

Demil B., Lecocq X., (2010), "Business Model Evolution: In search of dynamic Consistency”, Long Range Planning, 43, pp. 227-246

Detchessahar, M., Journé, B., Kessous, E., (2003), «Logistique et modèles d'affaires. L'exemple du commerce électronique », Revue Française de Gestion, n 143, pp. 95-110

Durand, B., (2008), «Les magasins de proximité : un atout logistique pour l'épicerie en ligne », La Revue des Sciences de Gestion, $\mathrm{n}^{\circ} 229$, pp. 75-83 
Dang Nguyen, G., Mevel, O., (2007), « Nouvelle et ancienne économie, vers une intégration réussie ? », Revue Française de Gestion, $\mathrm{n}^{\circ} 173$, pp. 113-130

Esper, T.L., Crook, T.R., (2014), «Supply Chain Resources: Advancing Theoretical Foundations and Constructs", Journal of Supply Chain Management, vol 50, n³, pp. 3-5

Esper, T.L., Jensen, T.D., Turnipseed, F.L., Burton, S., (2003), “The last mile : an examination of effects of online retail delivery strategies on consumers", Journal of business logistics, vol 24, n 2, pp. 177-203

Fabbe-Costes N, Paché G, (2013), La logistique, Une approche innovante des organisations, PUP

Field, J. M., Heim, G.R., Sinha, K.K., (2004), "Managing quality in the e-service system : development and application of a process model”, Production and Operations Management, vol 13, n4, pp. 291-306

Girotra K., Netessine S., (2014), "Four paths to business model innovation”, Harvard Business Review, July-August, pp. 96-103

Griffis, S.E., Rao S, Goldsby T.J., Voorhees, C.M, Iyengar, D., (2012), «Linking order fulfillment performance to referrals in online retailing : an empirical analysis », Journal of Business logistics, 33(4), pp. 279-294

Gunther McGrath R., (2010), “Business Models: A Discovery Driven Approach”, Long Range Planning, 43, pp. 247-261

Hantula, D. A., Bryant, K. , (2005), «Delay discounting determines delivery fees in an e-commerce simulation: a behavioral economic perspective », Psychology \& Marketing, vol 22(2), pp. 153-161

Huang, Y.K., Feng, C.M., Fan, W.S., Lin, H.P. (2012), "Exploring the choice behavior on the retailing delivery provider for online auction consumers", International Journal of Electronic Commerce Studies, vol 3, N², pp 325-334

Hult, T.M., Boyer, K.K., Ketchen, D. J. Jr, (2007), "Quality, operational logistics strategy, and repurchase intentions : a profile deviation analysis", Journal of business logistics, vol 28, n², pp. 105-132

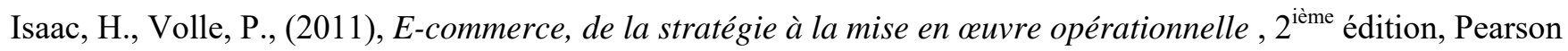

Isckia, T., (2009), "Amazon's evolving ecosystem: A cyber-bookstore and Application Service Provider », Canadian Journal of Administrative Sciences, Volume 26, Issue 4, pages 332-343

Itami, H. et Nishino, K, (2010), « Killing two birds with one stone », Long Range Planning, vol 43, pp. 364-369

Kessous, E., (2001), « Le commerce électronique et la continuité de la chaîne logistique », Réseaux, n 106, pp. 103-133

Kim, M.., Lennon, S., (2011), «Consumer response to online apparel stockouts », Psychology \& Marketing, vol 28(2), pp. $115-144$

Kokkinaki,, U., Dekker, R., Van Nunen, J., Pappis, C., (1999), "Etude exploratoire sur le commerce électronique lié à la reverse logistics", Logistique \& Management, vol. 7, n 2 , pp. 27-36

Kuk, G., Janssen, M., (2013), "Assembling infrastructures and business models for service design and innovation », Information Systems Journal, vol 23, pp. 445-469

Lambert, S. C., (2015), "The importance of classification to business model research”, Journal of Business Models, vol. 3, $\mathrm{N}^{\circ} 1$, pp. 49-61

Lambert, S. C., Davidson, R. A., (2013), « Applications of the business model in studies of enterprise success, innovation and classification : an analysis of empirical research from 1996 to 2010 », European Management Journal, vol. 31, pp. 668-681

Lecocq, X., Demil, B., Ventura, J., 2010, « Business Models as a research program in strategic management : an appraisal based on Lakatos »,M@n@gement,vol 13,n²,pp.214-225

Mentzer J.T., De Witt W.D., Keebler J.S., Min S., Nix, N.W, Smith C.D, Zacharia Z.G.,(2001), , « Defining supply chain management », Journal of Business Logistics, vol 22, n², pp 1-25

Marouseau, G., (2005), «Le système logistique, facteur-clé du succès des cybermarchés », Logistique \& Management, vol. $13, \mathrm{n}^{\circ} 2$, pp.9-19

Marouseau, G., (2001), « Modèles logistiques du commerce électronique », Logistique \& Management, vol. 9, $\mathrm{n}^{\circ}$ 1, pp. $41-50$

Moingeon, B. , Lehmann-Ortega, L.,(2010), «Genèse et déploiement d'un nouveau business model : l'étude d'un cas Désarmant»,M@n@gement, vol 13,n4,pp 266-297

Mollenkopf, D.A., Rabinovich, E., Laseter, T.M., Boye, r K. K., (2007), "Managing internet product returns: a focus on effective service operations", Decision Sciences, vol 38, number 2, pp. 215-240 
Morris, M., Schindehutte, M., Allen, J., (2005), «The entrepreneur's business model: toward a unified perspective », Journal of Business Research, vol 58, pp. 726-735

Osterwalder, A. (2004). The business model ontology: A proposition in a design science approach., thèse Présentée à l'Ecole des Hautes Etudes Commerciales de l'Université de Lausanne, http://www.uniempre.org.br/userfiles/files/TheBusiness-Model-Ontology.pdf

Osterwalder, A., Pigneur, Y., Tucci, C. L ., (2005), «Clarifying Business Models : origins, present, and future of the concept », Communication of the Association for Information Systems, vol. 15, May, pp 1-38

Osterwalder, A., Pigneur, Y., (2010), Business Model Generation, a handbook for visionaries, game changers and challengers, John Wiley \& Sons

Plé, L, Lecocq, X, Angot, J,(2010), «Customer-Integrated Business Models : A theoretical Framework », M@n@gement, vol 13, n 4, pp 226-265

Porter, M. E., (2001), « Strategy and the Internet », Harvard Business Review, March, pp. 62-78

Rabinovich, E., (2005), « Consumer direct fulfillment performance in internet retailing: emergency transshipments and demand dispersion », Journal of Business logistics, vol 26, $\mathrm{n}^{\circ} 1$, pp. 79-112

Rabinovich, E., (2004), "Internet retailing intermediation : a multilevel analysis of inventory liquidity and fulfillment guarantees", Journal of Busness logistics, vol 25, n², pp 139-169

Rabinovich, E., (2007), "Linking e-service quality and markups: the role of imperfect information in the supply chain", Journal of Operations Management, vol. 25, pp. 14-41

Rabinovich, E. et Evers P.T., (2003), "Product fulfillment in supply chains supporting internet-retailing operations", Journal of business logistics, vol 24, n², pp. 205-236

Rabinovich, E., Knemeyer, A. M., Mayer, C. M., (2007), "Why do internet commerce firms incorporate logistics service providers in their distribution channels? The role of transaction costs and network strength", Journal of Operation Management, vol 25, issue 3, pp. 661-681

Rabinovich, E.., Rungtusanatham, M., Laseter, T. M., (2008), "Physical distribution service performance and internet retailer margins: the drop-shipping context", Journal of Operations Management, vol 26, pp. 767-780

Rabinovitch, E., Sinha, R., Laseter, T., (2011), "Unlimited shelf space in internet supply chains : treasure trove or wasteland?", Journal of Operations Management, vol 29, pp. 305-317

Randal,1 T., Netessine, S., Rudi, N., (2006), "An empirical examination of the decision to invest in fulfillment capabilities: a study of internet retailers", Management Science, vol 52, n 4 , pp. 567-580

Rao, S., Goldsby, T.J., Griffis, S.E., Iyengar, D., (2011), "Electronic logistics service quality (e-LSQ) : its impact on the customer's purchase satisfaction and retention", Journal of business logistics, 32(2), pp. 167-179

Rao, S., Rabinovich, E., Raju, D., (2014), "The role of physical distribution services as determinants of product returns in internet retailing", Journal of Operations Management, vol 32, pp. 295-312

Rappa, M., (2010), "Business models on the Web”, http: // digitalenterprise.org/models/models_text.html

Rouquet, Goudarzi, Henriquez, (2010), «Le transfert d'activités logistiques entreprise-consommateur : une comparaison des cas Auchan Drive et IKEA », Logistique \& Management, vol. 18, n², pp. 49-61

Sanders, N. R., (2007), « The benefits of using e-business technology: the supplier perspective », Journal of Business Logistics, vol 28, pp. 177-207

Schneider S., Spieth P., (2013), "Business model innovation: towards an integrated future research agenda », International Journal of Innovation Management, vol 17, $\mathrm{n}^{\circ}$ 1, 1340001 (34 p.)

Taylor, J.C., Fawcett, S.E., Jackson, G.C., (2004), « Catalog retailer in-stock performance : an assessment of customer service levels », Journal of business logistics, vol 25, n², pp 119-137

Teece, D.J., (2010), “Business Models, Business Strategy and Innovation”, Long Range Planning vol 43, pp 172-194.

Timmers, P., (1998), "Business Models for Electronic Markets", CommerceNet, 9 Septembre 1998, Research Note \#9821

Tokman, M., Richey, R. G., Deitz, G. D., Adams, F. G., (2012), "The retailer's perspective on the link between logistical resources and perceived customer loyalty to manufacturer brands", Journal of Business Logistics, 33(3), pp. 181-195

Waller, M. A., Fawcett, S. E., (2013), "Data science, predictive analytics, and big data: a revolution that will transform supply chain design and management", Journal of Business Logistics, 34(2), pp. 77-84

(c) 2017 ISTE OpenScience - Published by ISTE Ltd. London, UK - openscience.fr 
Wang, Y-S., (2008), "Assessing e-commerce systems success: a respecification and validation of the DeLone and McLean model of IS success", Info Systems J vol. 18, pp. 529-557

Wirtz, B. W., Pistoia, A., Ullrich, S., Götte, 1 V., (2016), "Business Models : Origin, Development and Future Research Perspectives", Long Range Planning, vol 49, pp 36-54

Zott, C., Amit, R., Massa, L., (2011), "The Business Model : Recent Developemets and Future Research", Journal of Management, vol 37, n4, pp 1019-1042.

Zott, C., Amit, R., (2010), "Business Model Design : an Activity System Perspective”, Long Range Planning, vol 43, pp. 216-226 
Exemple : La Redoute

Elle dispose d'un entrepôt de $250000 \mathrm{~m} 2$ à la Martinoire (Wattrelos) avec $30 \mathrm{~km}$ de convoyeurs ; sur les 3000 collaborateurs du groupe, 1400 sont rattachés à la logistique (dont 300 pour les retours) ; pour le transport elle s'appuie sur sa filiale Relais Colis qui dispose elle-même de 800 véhicules (en leasing), 300 chauffeurs, 3 hubs nationaux, 26 agences régionales et associe 4000 commerces de proximité en points relais colis pour organiser 600 tournées quotidiennes.

Jusqu'à récemment, Telemarket (racheté par système $U$ pour pénétrer le marché parisien) intégrait sa logistique (entrepôt semi-automatisé à Pantin), mais également son transport avec une flotte de véhicules qui livraient en moyenne 14 clients par tournée (soit en moyenne $50 \mathrm{~km}$ ).

Il en est de même pour Place du marché (Toupargel), pour qui les livreurs ont un rôle commercial.

«24 heures Chez Vous» (coût 3,95 € en supplément des frais de livraison standard)

«Colissimo»" (2 à 4 jours pour la livraison, la livraison est faite par la poste)

«Relais Colis» (2-4 jours).

« Livraison sur rendez-vous » pour les grands produits; Les délais de livraison varient de 2 heures; Les frais de livraison varient selon le poids et la taille des produits

«Groupé port », si la commande comprend au moins trois produits spécifiques

Reprise de l'ancienne literie proposée de base et / ou matelas. La reprise est en même temps et au même endroit que la livraison. 1 produit retourné pour 1 produit livré. Coût: $25 € /$ produit (CGV)

Livraison à domicile

Offres commerciales comme la livraison gratuite dès 25 euros d'achats sur des créneaux d'une heure, à domicile (Toupargel)

17.000 Points de retrait gratuits à partir de $25 €$ d'achat pour les petits emballages ( $<$ de livraison de $30 \mathrm{~kg}$ par TNT Express points Cdiscount, "Relais Colis", "des points relais", "Chronopost", "SoColissimo", "abricolis"),

500 points de livraison gratuits à partir de $25 €$ d'achat pour les gros emballages;

Livraison gratuite (sur les nouveaux produits, et les consoles de jeux vidéo avec So Colissimo à la maison; sur tous les magasins à partir de $25 €$; pour les paniers de literie de $€ 249$ avec «livraison standard»; Sur des vins et spiritueux dès $150 €$ avec So Colissimo à la maison);

payant: à la maison pour les petits emballages (Colissimo, TNT Chez vous); 3 modes de LAD pour les grands produits.

Service d'abonnement annuel à 19 Euros. (CGV)

3 Suisses International a ouvert en 2013 le plus grand centre de préparation de commandes en France ; elle propose ses services aux autres ecommerçants par l'intermédiaire d'une filiale Dispeo ; 1200 personnes sont affectées à la logistique ; le groupe s'appuie sur sa filiale mondial relay et 4300 points relais en France ; le transport est par contre sous-traité et mondial relay est commissionnaire de transport.
Point de Relais ${ }^{\circledR}$ 48H gratuit; 4300 en France; forfait pour les frais de traitement des commandes (PFF, "Participation Forfaitaire aux frais" pour les éléments de tri, emballage,).

Livraison à domicile à $48 \mathrm{H}$ ou $72 \mathrm{H}$ Colissimo ${ }^{\circledR}$ (de la livraison de colis au bureau de poste), généralement une semaine après la confirmation de la commande.

Pour les colis qui ne respectent pas les normes de 
Pixmania a un hub automatisé en Essonne ; il propose également la prestation logistique aux autres e-commerçants mais sous-traite le transport à 27 transporteurs pour 26 pays.

Amazon aussi propose la prestation logistique (Amazon Fullfilment). prestation établies par la Poste, la livraison est assurée dans les mêmes conditions avec Mondial Relay (présenté comme un partenaire affilié).

Si la commande contient au moins un élément fragile ou lourde avec un PFF spécifique, dans ce cas, le client ne paie que la plus haute PFF.

Pour les petits paquets, le PFF est généralement $5.99 €$ (sous réserve de modification au cours de la saison), le PFF est GRATUIT pour Point Relais ${ }^{\circledR}$, pour toute commande d'un montant minimum de $49 €$, disponible en point Relais ${ }^{\circledR}$.

Pour les colis lourds ou volumineux, le montant de la PFF varie en fonction de l'article. Il peut être $19 €(\mathrm{~L} 1), 35 €(\mathrm{~L} 2)$ ou $59 €(\mathrm{~L} 3)$.

15 jours pour échanger ou de retourner un article, dans son emballage d'origine (CGV)

Amazon offre pas moins de 7 types de livraisons : livraison rapide, livraison rapide en point relais, livraison prioritaire, livraison en un jour ouvré, livraison au pied de l'immeuble, livraison dans la pièce désirée, livraison en soirée le jour même (Paris, Marseille, Lyon), livraison garantie le jour de la sortie. L'objectif est d'atteindre l'ensemble du territoire français ; le transport est offert à partir de 20 euros, plus rapide pour les formules premium (50 euros par an); une formule d'abonnement existe pour les consommables comme les couches (CGV). 
Type5 : différencié

géographiquement

et ouvert
Vente-privee .com a rapidement fait le choix d'intégrer la logistique, tout en s'appuyant sur des prestataires (pour les Etats-Unis par exemple). Concernant le transport, 95\% des commandes sont livrées par La Poste; les pondéreux sont pris en charge par des transporteurs privés.
« Pour comprendre les modalités de livraison de vente-privee.com, certains principes doivent être retenus lorsque vous passez une commande.

2 à 3 semaines après une vente, nous réceptionnons sur notre plateforme logistique les articles. Nous préparons ensuite votre commande et vous l'expédions soit via So Colissimo, via Relais Colis ou via transporteur spécialisé pour la France, et via DHL pour la Belgique. » (extrait des $\mathrm{CGV}$ )

« Les frais de livraison et de préparation varient selon :

- le poids, le volume, la fragilité de votre commande,

-le transporteur en charge de votre commande : So Colissimo, Relais Colis, Mondial Relay ou transporteur spécialisé,

- l'option de livraison choisie.

Vous pouvez visualiser les frais de livraison qui s'appliquent à votre commande dans votre panier avant le paiement de votre commande.

Lors d'une vente, si vous souhaitez compléter un premier panier déjà validé par des produits issus de la même vente, des frais de port additionnels s'ajouteront à ceux préalablement facturés. Leur montant vous sera communiqué avant de valider le paiement de votre second panier.

En revanche, les frais de port et de préparation logistique de deux commandes distinctes ne peuvent en aucun cas être regroupés » (extrait des CGV).

Delamaison offre tout type de produit destiné à la maison, de la cuillère au canapé en passant par les portes de jardin ; l'objectif est de livrer tous les articles en une fois, quelles que soient leurs caractéristiques. Cela génère cinq types de commandes à livrer : commandes mono-coli (petits/moyens ou lourds et volumineux), des commandes multi-colis (petits/moyens, lourds et volumineux ou multi-typologie). Même les produits de la dernière catégorie font l'objet d'une livraison unique, par un seul transporteur. Le transport est essentiellement de la livraison à domicile compte tenu des produits proposés. Le système d'information est capable d'identifier le transporteur le plus adéquat pour livrer la commande. La gestion de la fiche d'identité de l'article est un vecteur capital, de l'offre.

Delamaison a par ailleurs ouvert un Drive aux Ulysses, adossé à leur entrepôt ; c'est un transfert de charge intéressant pour le e-commerçant, car il s'est rendu compte que des clients étaient près à traverser Paris pour venir chercher leur produit.

Chronopost, colissimo, so colissimo ou mondial relay Kiala, pour Showroomprivé.
Type 7 : logistique intégrée par
Rakuten (e-commerçant japonais) a racheté Priceminister et ADS, ce qui lui permet d'ouvrir un 
croissance externe ou achat partiel

Type 8 : logistique

et transports soustraités mais

transports pilotés

Type 9 : logistique

et transport sous-

traités avec

mutualisation

prestée

(Brandalley) nouvel entrepôt pour les services logistiques lancés par les deux sociétés (Journal Du Net, 4.2.2013).

Showroomprivé.com est passé par un site de Deret pour sa logistique, qu'il a intégré par le biais d'une société commune détenue à parts égales avec

Deret ; d'une logistique principalement interne, il est passé à une logistique plutôt externalisée. Les colis sont ensuite injectés dans les hubs européens des transporteurs (et facturés au poids). La Poste a un rôle prépondérant.

Pour la rueducommerce.com, contrairement aux achats et à la relation fournisseur, la logistique ne fait pas partie du comité de direction ; elle est soustraitée et opère à partir d'un entrepôt de $20000 \mathrm{~m} 2$ (24 jours de couverture en 2008) proche de Lyon ; le transport lui aussi est sous-traité ; c'est néanmoins larueducommerce.com qui choisit ses transporteurs (5 transporteurs mais 59\% des transports réalisés par la Poste et Chronopost).
« Showroomprive.com se réserve le droit de pratiquer des offres tarifaires sur les frais de port associés aux commandes » (CGV).

Livraison rapide ou express en point relais ou à domicile, gratuite à partir d'un certains montant d'achat (CGV).
Brandalley utilise pour sa logistique une chaîne de tri automatisée installée par Sed Logistique dans le Val d'Oise, mutualisée avec Jennyfer; elle passe par Kiala, Chronopost, Colizen (pour Paris) et Colissimo pour le transport.
La société Brandalley5 a une politique du panier unique quel que soit le nombre de « ventes privées » : le client a une livraison, une fois les frais de port à payer même s'il achète des produits de plusieurs marques (et donc plusieurs ventes) ; côté préparation, cela suppose qu'on attende la dernière pièce du panier avant de déclencher l'expédition et donc qu'on allonge les délais au profit d'une commande consolidée. Cependant, pour les pièces disponibles en stock (collections en cours, collections passées mais pas ventes privées), les délais de livraison sont de 6 heures à Paris et en région parisienne (avec Colizen), 24 heures en province en passant par Chronopost ou Chrono Relais et 48 heures en passant par Colissimo. 55\% des colis sont expédiés en 24 heures et $95 \%$ en moins de trois semaines. Concernant les retours, la politique du ecommerçant est de les faciliter sans les encourager ; les frais sont donc à la charge du client si le client est à l'origine du retour mais à la charge de Brandalley dans le cas contraire. Le client bénéficie néanmoins d'un retour gratuit en cas de demande d'échange ou en cas de remboursement par avoir (ce qui leur permet une facilité de trésorerie). Environs 10\% des produits commandés fait l'objet d'un retour; dans $14 \%$ des cas c'est du fait de Brandalley. Pour le responsable logistique, la logistique est une arme marketing (conférence sur le e-commerce, Paris, 7 décembre 2012).

Tableau. Exemples pour chaque type identifié

${ }^{5}$ Le directeur logistique est aussi responsable du service client chez Brandalley, ce qui montre l'importance de cette fonction pour le e-commerçant et son intégration stratégique dans un processus orienté client. 